\title{
Dense Polytetrafluoroethylene Membrane versus Titanium Mesh in Vertical Ridge Augmentation: Clinical and Histological Results of a Split-mouth Prospective Study
}

\author{
Carlo Maiorana ${ }^{1}$, Filippo Fontana ${ }^{2}$, Marco Rasia dal Polo ${ }^{3}$, Stefano Pieroni ${ }^{4}$, Luca Ferrantino ${ }^{5}$, Pier Paolo Poli ${ }^{6}$, \\ Massimo Simion ${ }^{7}$
}

\begin{abstract}
Aim and objective: The aim of the present split-mouth prospective study was to evaluate clinically and histologically the bone regeneration obtained following preprosthetic vertical bone augmentation performed with titanium-reinforced dense polytetrafluoroethylene membrane (d-PM) compared to titanium mesh (TM).

Materials and methods: Healthy adult patients presenting with bilateral partial edentulism in the posterior mandible requiring vertical ridge augmentation for implant placement purposes were consecutively included. One side of the mandible was randomly assigned to the use of $\mathrm{d}$-PM, the other to TM. The graft consisted in a mixture of autogenous bone harvested nearby the surgical site and deproteinized bovine bone mineral particles in a 1:1 ratio. On each side during bone augmentation surgery, a 2-mm diameter mini-implant was inserted for clinical and histological analyses. After a healing period of 8 months, the second surgical phase was carried out to remove the nonresorbable barriers, to evaluate clinically the vertical bone gain, and to collect a bone biopsy that included the mini-implant. During the same surgical session, dental implants were inserted in a prosthetically guided position.

Results: A total of five patients were enrolled. Eight out of 10 sites healed uneventfully. In the remaining two sites, premature exposure of the TM was observed. Mean vertical bone gain of 4.2 and $1.5 \mathrm{~mm}$ was achieved in d-PM and TM groups, respectively $(p=0.06)$. A mean mineralized tissue of 48.28 and $35.54 \%$ was observed in d-PM and TM groups, respectively $(p=0.51)$.

Conclusion: The vertical bone gain, although not significantly, was higher in the d-PM group. Similar histological outcomes were noticed if exposure did not occur. In case of wound dehiscence, major resorption was observed.

Clinical significance: Both d-PM and TM can be used to augment atrophic localized ridges vertically. The outcome of bone regeneration seems to be impaired by exposure of the device.

Keywords: Bone atrophy, Bone regeneration, Dental implants, Histology, Nonresorbable membranes, Prospective cohort study, Titanium mesh. The Journal of Contemporary Dental Practice (2021): 10.5005/jp-journals-10024-3091
\end{abstract}

\section{INTRODUCTION}

Vertical bone loss in partially edentulous patients represents a major challenge in implant dentistry due to anatomical limitations and technical difficulties. Regeneration of vertical bone defects has been advocated to allow proper implant placement avoiding unfavorable crown-to-implant ratio and to obtain a better aesthetic outcome. Different surgical procedures have been proposed to handle alveolar bone atrophies, including guided bone regeneration (GBR), onlay and/or inlay bone grafting, alveolar distraction osteogenesis, and titanium meshes. ${ }^{1-4}$ However, there is inconclusive evidence to support the superiority of a surgical technique over another.,

GBR with nonresorbable membranes has proved its effectiveness in obtaining vertical augmentation with stable longterm results. ${ }^{7,8}$ The rationale of GBR is the use of a cell-occlusive barrier to promote undisturbed healing of the underlying bone graft. Further essential characteristics of nonresorbable membranes such as biocompatibility, space-making abilities, tissue integration, and easy handling have been fulfilled by polytetrafluoroethylene (PTFE) devices. In the last years, dense PTFE membranes have demonstrated clinically and histologically their efficacy during bone augmentation procedures. ${ }^{9,10}$

On the other hand, the use of titanium meshes for the reconstruction of osseous defects has been firstly proposed in maxillofacial surgery and then applied in the treatment of deficient
1-4,6Implant Center for Edentulism and Jawbone Atrophies, Maxillofacial Surgery and Odontostomatology Unit, Fondazione IRCCS Cà Granda Ospedale Maggiore Policlinico, University of Milan, Milan, Italy

${ }^{5}$ Department of Aesthetic Dentistry, Istituto Stomatologico Italiano, University of Milan, Milan, Italy

${ }^{7}$ Department of Periodontology, Maxillofacial Surgery and Odontostomatology Unit, Fondazione IRCCS Cà Granda Ospedale Maggiore Policlinico, University of Milan, Milan, Italy

Corresponding Author: Pier Paolo Poli, Implant Center for Edentulism and Jawbone Atrophies, Maxillofacial Surgery and Odontostomatology Unit, Fondazione IRCCS Cà Granda Ospedale Maggiore Policlinico, University of Milan, Milan, Italy, Phone: +390255032621, e-mail: pierpaolo_poli@fastwebnet.it

How to cite this article: Maiorana C, Fontana F, Rasia dal Polo M, et al. Dense Polytetrafluoroethylene Membrane versus Titanium Mesh in Vertical Ridge Augmentation: Clinical and Histological Results of a Splitmouth Prospective Study. J Contemp Dent Pract 2021;22(5):465-472.

Source of support: Nil

Conflict of interest: None

edentulous maxillary ridges. ${ }^{11-14}$ Several authors reported the use of titanium grids in association with a bone graft prior to or at the time of implant insertion. ${ }^{15-17}$ Extraoral or intraoral autogenous 
bone eventually combined with deproteinized bovine bone mineral (DBBM) particles, which are the most commonly used grafts. ${ }^{14,17-20}$ In the latter, the combination of an osteoconductive scaffold with intraorally harvested bone allows reducing the amount of autogenous bone needed to reconstruct the defect, thus reducing the postoperative patient morbidity. ${ }^{15}$

Interestingly, a direct comparison between PTFE membranes and titanium meshes is scarcely reported so far. Cucchi and coworkers compared dense PTFE membranes with titanium meshes covered by cross-linked collagen membranes in two groups of patients requiring vertical bone augmentation. Both approaches yielded nonstatistically significant differences in terms of complications, implant stability, and vertical bone gain from clinical, histological, and histomorphometric outcomes. ${ }^{21,22}$ Although these trials supported the use of both PTFE membranes and titanium meshes for bone regeneration, these two techniques have never been compared in a split-mouth clinical investigation. Therefore, the aim of the present split-mouth study was to clinically and histologically compare titanium-reinforced d-PTFE membranes to titanium meshes in preprosthetic vertical bone augmentation procedures.

\section{Materials and Methods}

\section{Study Design}

The present study was designed and conducted as a prospective spiltmouth clinical trial aimed to compare two different nonresorbable membranes used in vertical bone augmentation prior to implant insertion. In particular, the null hypothesis was that no difference would be found in the clinical and histological outcomes following bone regeneration obtained with d-PTFE membrane (d-PM) versus titanium mesh (TM) in partially edentulous mandibles.

The study protocol was submitted to and approved by the local institutional review board, with the registration number 293/12. The investigation was carried out according to the principles outlined in the Declaration of Helsinki on experimentation involving human subjects as revised in 2013. All patients received an exhaustive explanation of the surgical procedures, the possible associated risks, and the alternative prosthetic solutions. All subjects gave their informed consent for inclusion before they participated in the study.

\section{Study Population}

Subjects eligible for the study were screened and recruited at the authors' department according to defined inclusion and exclusion criteria. To be enrolled, patients had to present with bilateral posterior mandibular partial edentulism according to Applegate-Kennedy Class I, requiring vertical ridge augmentation to allow prosthetically guided implant placement (Fig. 1). Surgical correction of the alveolar ridge atrophy was needed to improve hard and soft tissue implant support, crown-to-implant ratio, and the final aesthetics of the definitive implant-supported fixed prosthesis. Patients selection was done according to the following inclusion criteria: (1) good general health; (2) vertical bone defect $>3 \mathrm{~mm}$ as measured on a panoramic radiograph, calculated as the distance between the deepest area of the edentulous ridge and the distal bone peak of the adjacent tooth in reference to the virtual occlusal plane; (3) interarch distance $>10 \mathrm{~mm}$; (4) age $\geq 18$ years; (5) no relevant medical conditions; (6) no medical history of head and neck radiation therapy; (7) no daily income of the following drugs: anticoagulants, antiplatelets, bisphosphonates, and glucocorticoids; (8) women neither pregnant nor lactating; (9) no smoking habits or smoking <10 cigarettes/day; (10) no
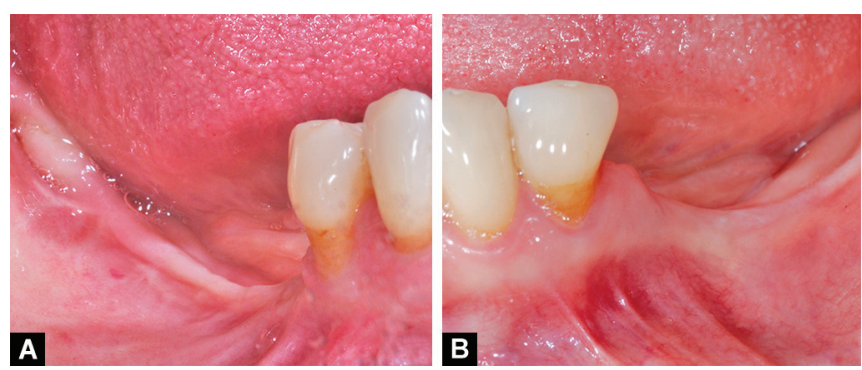

Figs $1 \mathrm{~A}$ and $\mathrm{B}$ : Intraoral clinical view of a patient showing bilateral partial edentulism requiring vertical bone augmentation: (A) Preoperative lateral view of the right side; (B) Preoperative lateral view of the left side

heavy bruxism; (11) no medical history of former teeth extractions involving the surgical sites in the previous 2 months; (12) Full Mouth Plaque Score and Full Mouth Bleeding Score $<25 \%$ assessed at four sites per remaining tooth ${ }^{23}$; and (13) no active periodontal disease.

\section{Materials}

The d-PM consisted of a nonresorbable high-density titaniumreinforced polytetrafluoroethylene membrane with a porosity size of less than $0.3 \mu \mathrm{m}$ made of two d-PTFE layers with an interposed titanium structure (Cytoplast, Osteogenics Biomedical Inc., Lubbock, Texas, USA). The TM consisted of a grade I titanium grid characterized by a thickness of $0.12 \mathrm{~mm}$ and holes of $0.5 \mathrm{~mm}$ (AON Implant, Vicenza, Italy).

\section{Randomization}

In each subject, the surgical site was allocated to either d-PM or TM group according to a block randomization created on a Web-based program (Sealed Envelope Ltd.). In brief, each patient was given an enrolment number and related surgical sites were given an identification code. At this point, surgical sites were randomly assigned to either the d-PM or TM group according to the sequentially numbered, sealed envelope protocol using the computer spreadsheet. In order to avoid bias, the individually assigned sealed envelopes were opened after tenting screw positioning and first clinical measurement. Only one collaborator not involved in the study was aware of the random sequence and could have access to the randomization list. A copy of the randomization sequence was preserved for assessment at the end of the study.

\section{Interventions}

Each patient was treated according to a previously described surgical protocol for a two-stage vertical ridge augmentation. ${ }^{24} \mathrm{All}$ surgical procedures were performed on an outpatient basis under local anesthesia under sterile conditions by the same surgical team.

Each site underwent three surgeries: (1) vertical ridge augmentation procedure; (2) d-PM/TM removal, sampling of a trephine bone block containing the mini-implant, and implant placement; and (3) implant uncovering and healing abutment connection.

After having entered into the study, each patient underwent second-level radiographic examination by means of computed tomography scan of the edentulous area to assess the morphology of the alveolar ridge.

Before starting the surgical procedure, preoperative rinsing of the oral cavity with chlorhexidine digluconate $0.2 \%$ antiseptic solution (Corsodyl, GlaxoSmithKline SpA, Verona, Italy) for 
2 minutes and perioral skin disinfection with povidone-iodine $10 \%$ aqueous solution (Betadine, Meda Pharma SpA, Milan, Italy) were performed. Intramuscular injections of $4 \mathrm{mg} / \mathrm{mL}$ of dexamethasone sodium phosphate (Soldesam, Laboratorio Farmacologico Milanese, Varese, Italy) were performed to reduce postoperative edema. A sedative premedication with oral diazepam $0.2 \mathrm{mg} / \mathrm{kg}$ (Valium, Roche SpA, Monza, Italy) was administered 30 minutes before surgery.

Local anesthesia was induced with infiltrations of articaine $4 \%$ with epinephrine 1:100.000 (Citocartin, Molteni Dental Srl, Milan, Italy). A full-thickness flap was elevated from the distal remaining tooth to the mandibular ramus, continuing in the sulcus of one or two mesially located teeth. Two vertical releasing incisions were performed at the distal and mesial ends of the crestal incision. After lingual and buccal flap elevation and passivation, a stainless-steel tenting screw (Dental Implant System, ACE Surgical Supply Co., Inc., Brockton, Massachusetts, USA) was used to ensure the device stability in maintaining the vertical dimension. At the same time, a mini-implant of $2 \mathrm{~mm}$ in diameter and $8-10 \mathrm{~mm}$ in length (AoN Implants Srl, Vicenza, Italy) was inserted. Both the tenting screw and the mini-implant were left to protrude from the alveolar crest depending on the required vertical regeneration. The distance between the top of the implant head and the first visible boneto-implant contact was assessed at the mesial and distal aspect of each mini-implant with a periodontal probe (PCP-UNC 15, Hu-Friedy, Chicago, Illinois, USA) for subsequent clinical evaluations of the vertical bone gain (Fig. 2). At this point, the treated site was randomly allocated to either the d-PM or TM group according to the randomization list. The contralateral site was generally treated after 2 months. The bone graft consisted in a mixture of autogenous bone chips harvested from the retromolar region by means of a bone scraper (Safescraper, Meta, Reggio Emilia, Italy) and deproteinized bovine bone mineral particles (Bio-Oss, Geistlich Pharma AG, Wolhusen, Switzerland) in a 1:1 ratio. Multiple perforations of the cortical bone were done to ensure blood supply. The assigned device was then fixed both lingually and buccally with osteosynthesis screws (Omnia Srl, Parma, Italy) (Fig. 3). Mucoperiosteal flaps were checked for proper passivation, and a first intention healing of the surgical wound was accomplished with horizontal mattresses and single stitches.

All patients underwent antibiotic treatment consisting of $1 \mathrm{gm}$ of amoxicillin clavulanate (Augmentin, GlaxoSmithKline, Verona, Italy) three times daily for 10 days starting 2 days before the surgical procedure. Oral ketoprofen 80 mg tablet (Oki, Dompé Farmaceutici
SpA, Milan, Italy) was prescribed for pain relief 1 hour before surgery and three times daily for 4 days. Patients were also instructed to rinse with a $0.2 \%$ chlorhexidine digluconate solution twice daily up to suture removal. Postoperative instructions were given including cold pack applications, soft diet, and no demanding physical work or exercise. Sutures were removed 14 days after surgery. During the healing period, none of the patients were provided with provisional prosthesis because surgeries did not involve any aesthetic area and to reduce the risk of wound dehiscence at the regenerated site.

After 8 months of submerged healing at each site, all patients underwent the second surgical phase in order to remove the nonresorbable barrier, the space-maintaining mini-screw, and to harvest the bone sample, which included the mini-implant by means of a 4-mm diameter trephine. Conical screw-shaped dental implants with an abrasive-blasted and acid-etched surface (CONELOG Implant System, CAMLOG Biotechnologies GmbH, Basel, Switzerland) were subsequently placed in a prosthetically guided position. Implant lengths and diameters were progressively decided according to the residual ridge dimensions.

Healing abutments were connected to the implants 4 months after implant insertion. Temporary acrylic resin prostheses were

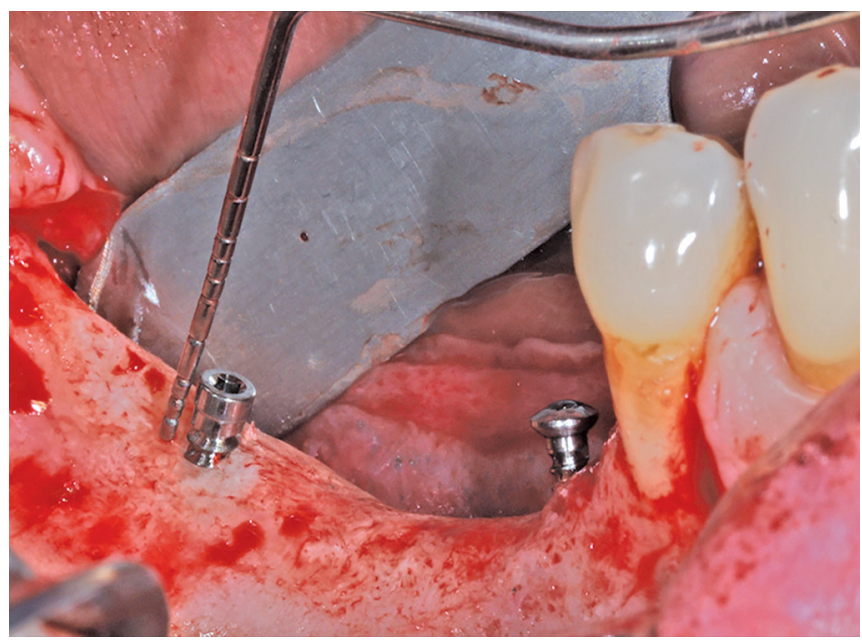

Fig. 2: Intrasurgical lateral view. Tenting screw and mini-implant are inserted in the bone defect. The periodontal probe is visible at the distal aspect of the mini-implant, measuring the distance between the top of the implant head and the first visible bone-to-implant contact
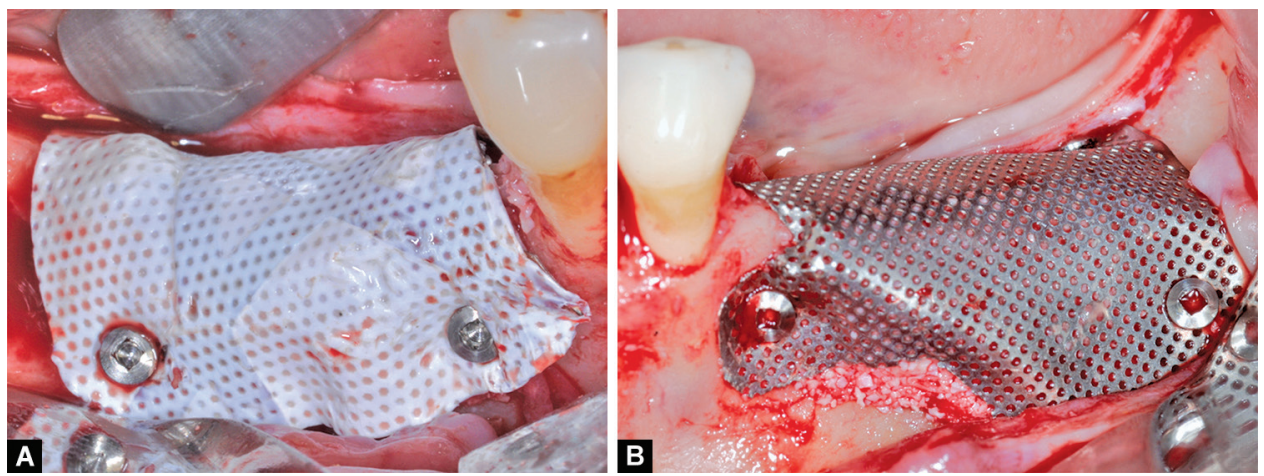

Figs $3 A$ and $B$ : Intrasurgical lateral view following grafting of the bone defect: (A) d-PM secured in the proper position by means of osteosynthesis screws; (B) TM fixed to the underlying bone with osteosynthesis screws 
delivered 1 month after healing abutment connection. Cementedretained prosthetic rehabilitations were finalized 3 months later. All patients were finally placed on an individually tailored professional maintenance program.

\section{Patient Records and Clinical Outcomes}

At the first visit and during the entire study period, all data were recorded on a patient form by a single clinical examiner, to avoid any involvement between the surgeon and the patient in the pre- and postoperative assessments. The following data were initially recorded: name, gender, age, site of surgery, and smoking habits. After the first surgical phase, the patients were followed for clinical evaluation every week for the first month and once a month thereafter. The presence of edema, hematoma, suppuration, flap dehiscence, flap necrosis, anesthesia, paresthesia, and dysesthesia was evaluated and, eventually, reported on the patient form. The distance between the top of the mini-implant head and the first visible bone-to-implant contact (implant head-bone level, Ih-BI) assessed at the mesial and distal aspect of each mini-implant was recorded at the time of bone regeneration procedure and at the reentry surgery. Values were rounded to the nearest millimeter. The outcome variable was the amount of regenerated vertical bone gain (VBG), obtained by subtracting the lh-Bl recorded at reentry surgery to the Ih-BI measured at augmentation procedure. The radiographic assessment consisted of periapical X-rays or orthopantomographs taken at baseline, after the first surgery, after implant insertion, after healing abutment connection, and 1 year after the final prosthetic restoration.

\section{Histologic and Histomorphometric Analysis}

The retrieved specimens were dehydrated in ethanol and then infiltrated in glycolmethacrylate resin from a starting solution of $50 \%$ ethanol/resin and subsequently $100 \%$ resin. Each step in this process required a 24-hour period. The photopolymerization was achieved using a 48-hour blue light exposure, thus obtaining the resin blocks. After polymerization, the blocks were grounded to remove the excess of resin and expose the tissue and then glued on plastic slides using a methacrylate-based glue. A high-speed rotating blade microtome mounted on a precision cutting machine (Micromet, Remet SAS, Bologna, Italy) was used to separate the section from the block, obtaining a $250 \mu \mathrm{m}$-thick section. The section was then ground down to about $40 \mu \mathrm{m}$ using a grinding machine (LS-2A, Remet SAS, Bologna, Italy) equipped with waterproof grinding paper. After the grinding procedure was finished, each section was polished with polishing paper and $3 \mu \mathrm{m}$ polishing cream. Two different staining procedures were used for these sections. The toluidine blue staining was used to analyze the different timing and remodeling pattern of the bone; the basic fuchsin staining was used to distinguish the fibrous tissue by exploiting an improved contrast.

The morphometric analysis was performed by digitalizing the images from the microscope with a Color Video Camera (JVC TK-C1380, JVC, Yokohama, Japan) and a frame grabber. Subsequently, the digitalized images were analyzed using image analysis software (ImageJ, National Institutes of Health, Bethesda, Maryland, USA). The images of the sectioned implant and the surrounding bone were acquired with magnifications ranging from $5 \times$ to $50 \times$.

For each retrieved specimen, the area of vertical regeneration was manually identified with a mouse cursor as the region of interest (ROI). Within the ROI, the proportion occupied by different tissues was assessed considering the following categories: mineralized tissue, nonmineralized tissue, and residual DBBM. In addition, boneto-implant contact (BIC), defined as the percentage of the implant surface directly contacted by mineralized tissue, was calculated. For this purpose, only the implant surface adjacent to the ROI was considered.

\section{Statistical Analysis}

Each surgical site was considered as a statistical unit. All variables were reported as mean, standard deviation, and range. A preliminary Shapiro-Wilk test was used to assess the normality of data distribution of each parameter. Student's paired $t$-test was used to compare normally distributed variables, whereas Wilcoxon signedrank test was applied for those variables that proved not to be normally distributed. All analyses were performed using statistical software (Stata 11, StataCorp LLC, College Station, Texas, USA). $p$ values of $p<0.05$ were considered to be statistically significant.

\section{Results \\ Clinical Results}

Five consecutive patients received vertical augmentation procedures performed with d-PM and TM according to a randomized split-mouth approach giving a total of 10 surgical sites. Patients were one male and four females, with a mean age of $54.2 \pm 12.5$ years (age range: $38-65$ ). None of the patients had smoking habits. No dropouts occurred during the entire period of the study. Therefore, all patients completed the study and were available for the final evaluation.

No complications were reported during the 8-month healing period for three out of five patients ( 8 out of 10 surgical sites). All sites treated in the d-PM group and three sites treated in the TM group healed uneventfully. The remaining two sites treated in the TM group experienced premature exposure of the titanium grid, 7 weeks and 2 months postsurgery, respectively. In both situations, the TM was left in place and the patient was instructed to apply $0.2 \%$ chlorhexidine digluconate gel twice a day as suggested by a previous literature review. ${ }^{25}$ Weekly follow-up was mandatory. In one site, overinfection was observed. The patient was prescribed amoxicillin clavulanate $1 \mathrm{gm}$ three times a day for 7 days and the infection disappeared. In both cases, the TM was left in place and the area was left to heal spontaneously for the subsequent months according to the protocol timing without any further complication.

Considering the $\mathrm{Ih}-\mathrm{BI}$ distance at baseline, no statistically relevant differences were found between the d-PM group $(4.2 \pm 1.7 \mathrm{~mm})$ and the TM group $(3.4 \pm 1 \mathrm{~mm})(p=0.22)$. At the reentry surgery, a regenerated newly formed bone-like tissue was visible extending to the top of the mini-implant head in all sites treated in the $\mathrm{d}-\mathrm{PM}$ group, resulting in Ih-BI distance of $-0.05 \pm 0.6 \mathrm{~mm}$ (Fig. 4A). In the TM group, only two out of five sites presented the top of the mini-implant covered by regenerated newly formed hard tissue (Fig. 4B). In the remaining three sites, a residual vertical defect was still detectable, with consequent $\mathrm{lh}-\mathrm{BI}$ distance of $1.9 \pm 1.5 \mathrm{~mm}$. The statistical difference at reentry surgery between the two groups was not significative $(p=0.06)$. Overall, the vertical bone gain was $4.2 \pm 2.2 \mathrm{~mm}$ for the $\mathrm{d}$-PM group and $1.5 \pm 1.6 \mathrm{~mm}$ for the TM group. This difference was not statistically significant $(p=0.10)$. After 8 months during the second surgical phase, patients received a total of 21 dental implants placed in 

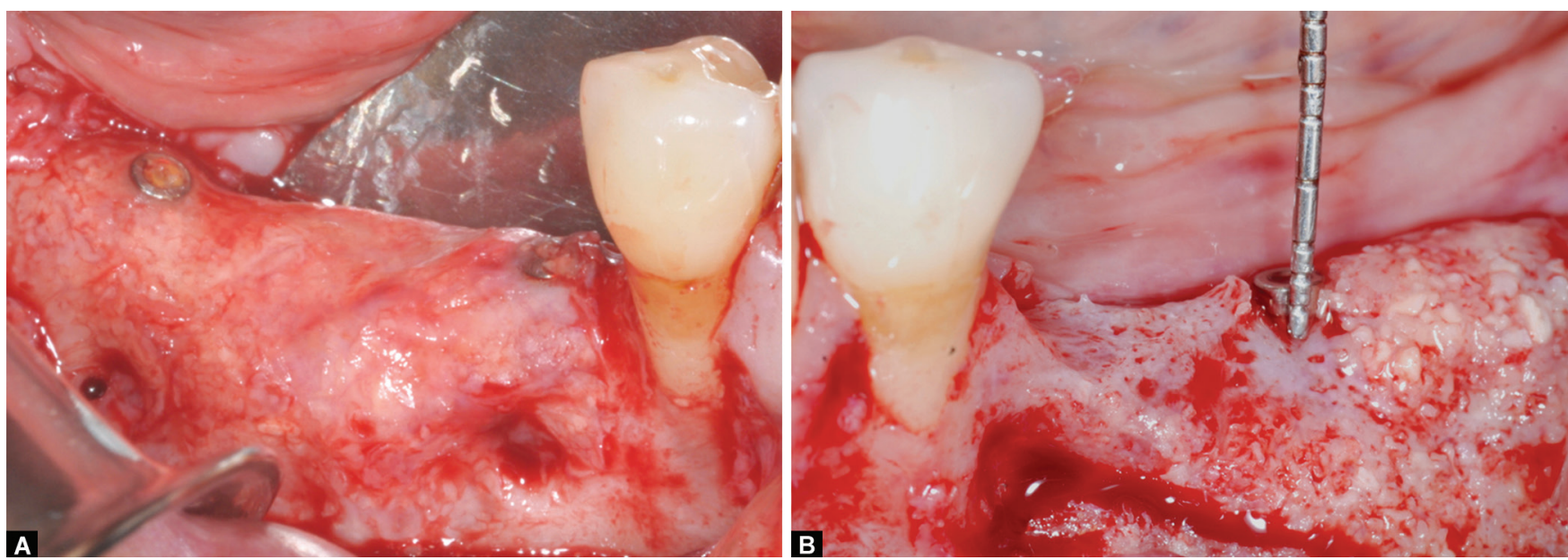

Figs 4A and B: Clinical outcome at the reentry phase following removal of the nonresorbable devices: (A) Lateral view of the d-PM side showing the mini-implant surrounded by newly formed bone-like tissue; (B) Lateral view of the TM side showing clinical measurement of the vertical bone gain around the mini-implant
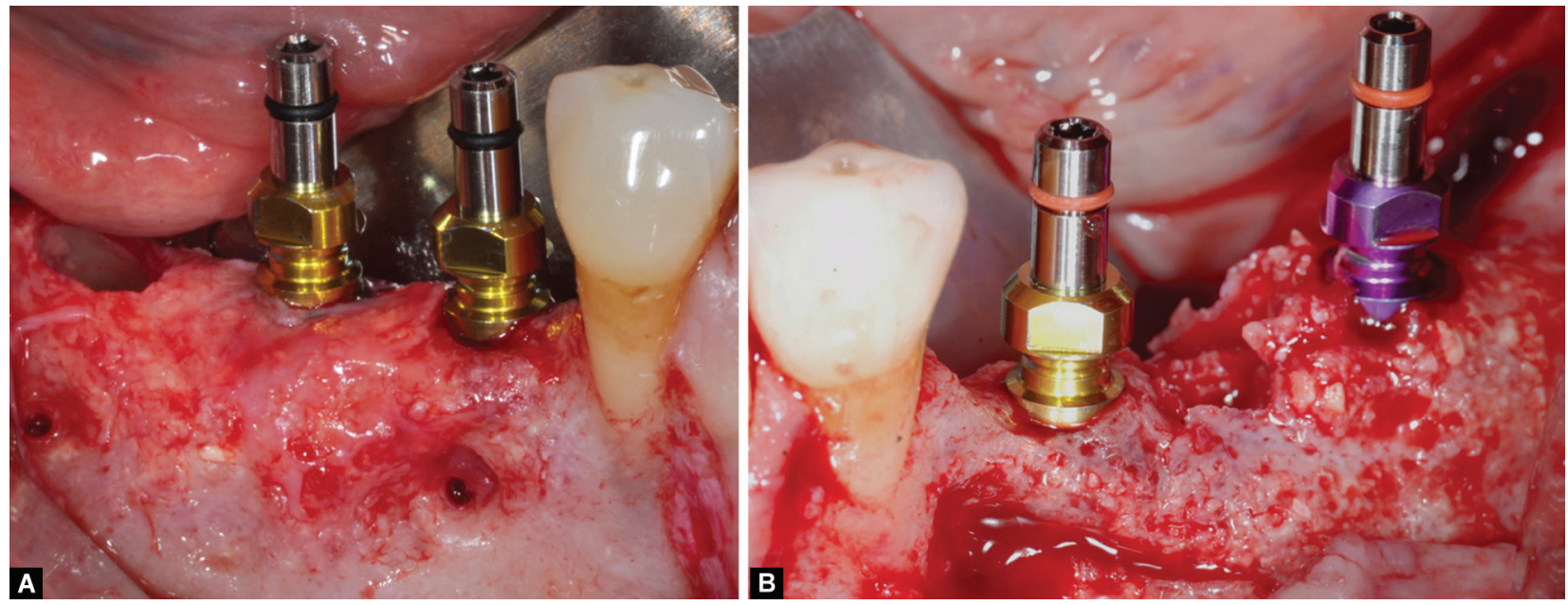

Figs 5A and B: Implant insertion during the reentry phase following bone biopsy: (A) Lateral view of the d-PM side showing implant placement; (B) Lateral view of the TM side showing implant placement

vertically augmented bone. A total of 11 implants were inserted in the d-PM group (Fig. 5A), while 10 were inserted in the TM group (Fig. 5B). At abutment connection, all implants appeared clinically stable and were therefore loaded with provisional and definitive implant-supported prosthetic restorations.

\section{Histologic Findings}

Out of 10 sites, a total of nine specimens were available for the histological analysis. One bone biopsy was severely damaged during the histological procedures and was therefore excluded from the histologic evaluation. Vertical bone regeneration was evident in both d-PM and TM groups (Figs 6A and B). Most samples demonstrated trabecular bone with different rates of maturation and mineralization in the regenerated area. Apical to the ROI, native lamellar bone was evident in direct continuity with the overlying regenerated bone. The ROIs presented different quantities of mature mineralized tissue, nonmineralized tissue, and DBBM particles. Mean mineralized tissue was $48.28 \%$ in the d-PM group and $35.54 \%$ in the TM group, with no statistically significant difference $(p=0.51)$. Nonmineralized tissue showed similar values in both groups, namely $29.92 \%$ in the d-PM group and $32.73 \%$ in the TM group without reaching statistical significance $(p=0.86)$. Mean percentage of DBBM particles was $19.31 \%$ in the d-PM group and $11.73 \%$ in the TM group. This difference was not statistically significant $(p=0.30)$. Into the ROI, bone marrow spaces including blood vessels were more frequently observed, if compared with those in the native bone area. Some specimens revealed a connective tissue layer in the most coronal part of the biopsy. In consideration of the two sites that showed exposure of the titanium grid, none or very limited bone regeneration was detectable (Fig. 6C). In most of the biopsies, the presence of residual DBBM granules was detected. These particles were generally surrounded by a layer of newly formed bone.

Generally, a variable amount of newly formed bone appeared in intimate contact with the implant surface (Fig. 6D). The mean BIC in the d-PM group was $53.85 \pm 40.40 \%$, while in the TM group the mean BIC was $28.5 \pm 20.34 \%$. This difference was not statistically significant between the two groups $(p=0.51)$. 


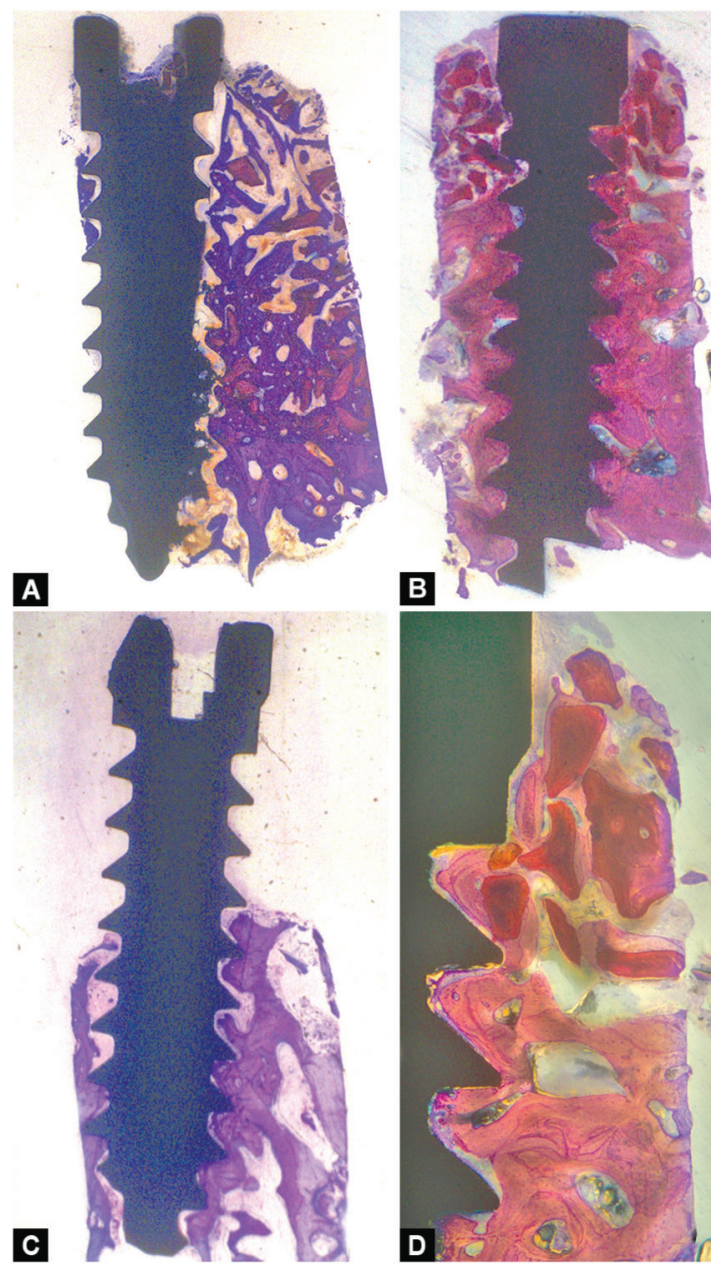

Figs 6A to D: Histological evaluation: (A) histological analysis from d-PM side showing the mini-implant and the surrounding bone at a magnification $5 \times$, toluidine blue stain; (B) human histological specimen from TM side; (C) histological analysis showing the mini-implant and the surrounding bone at a magnification $5 \times$, toluidine blue stain. No traces of newly formed tissue are detectable coronally to the native bone as a possible direct consequence of TM exposure; (D) histological analysis showing the mini-implant and the surrounding bone at a magnification $50 x$, basic fuchsin stain. The section shows the interface between the augmented area and the implant surface. The amount of newly formed bone within the threads is noteworthy. Remnants of DBBM surrounded by osteoid are still visible

\section{Discussion}

The purpose of the present study was to evaluate the clinical and histological outcomes of vertical ridge augmentation in posterior atrophic mandibles using d-PM or TM combined with a graft consisting of autogenous bone particles and DBBM granules. Overall, five patients with a bilateral partial edentulism were enrolled in the study, providing a total of 10 surgical sites. In each patient, the sites were randomly assigned to the d-PM or TM group.

The first issue of the present study referred to bone gain. Vertical bone regeneration was evaluated by means of a linear measurement in a coronal-apical direction around the miniimplant. This method has some limitations related to linear measurements. Indeed, a volumetric analysis would have probably revealed more precisely the amount of augmented bone. However, it must be noted that the methodology adopted herein has been widely validated in previous studies. ${ }^{7,10,24}$ Clinical results demonstrated that both techniques were successful in achieving bone growth. No statistically significant differences were observed when comparing the Ih-BI distance between the two groups in case of uneventful healing. A mean Ih-BI distance in the d-PM group of $4.20 \mathrm{~mm}$ is in accordance with previous clinical and histological studies. ${ }^{24,26}$ In a systematic review, the authors reported a vertical bone gain ranging from 2 to $8 \mathrm{~mm}$ when GBR procedures were carried out by means of PTFE membranes. ${ }^{5}$ Opposed to these findings, the mean vertical bone gain of $1.5 \mathrm{~mm}$ observed in the TM group was lower if compared to what is reported in the current literature. Roccuzzo et al. obtained a vertical bone fill of $4.8 \mathrm{~mm}$ ranging from 4 to $7 \mathrm{~mm}$, when titanium grids were used in association with autogenous bone. ${ }^{19}$ Results up to $8.6 \mathrm{~mm}$ were recently reported in a systematic review. ${ }^{27} \mathrm{~A}$ possible explanation of this difference is the incomplete bone fill that was noticed in case of TM exposure. In the two sites showing exposure of the TM, vertical bone gain varied from 0 to $0.5 \mathrm{~mm}$. This suggests that TM exposure could negatively affect the amount of newly formed bone underneath the barrier. As a matter of fact, by excluding both cases showing TM exposure, the average bone gain would have been $82 \%$ instead of $53 \%$ in the TM group. This finding further strengthens the healing behavior rather than the type of barrier as a crucial variable able to influence the outcome of the augmentation procedure.

The second issue of the present study referred to the complication rate. Premature exposure of nonresorbable membranes, which may compromise the final outcome of the regeneration, is considered the most common complication. ${ }^{28}$ In the present study, membrane exposure occurred in two out of five sites in the TM group. Conversely, in the d-PM group, healing proceeded uneventfully with no complications during the 8-month waiting time. A high complication rate following vertical bone augmentation procedures with nonresorbable membranes can be found in the literature. In particular, barrier device exposure may range between 0 and $45 \%$ for PTFE membranes and between 12 and 50\% for titanium meshes., 20 It is worth noting, however, that in the present study a significant discrepancy between the two groups has been observed. This is in accordance with a previous study showing a larger number of healing complications at sites treated with titanium meshes compared to sites treated with PTFE membranes. ${ }^{21}$ Drawing a possible explanation is complicated, since the same surgical approach has been adopted with both types of membranes. It may be speculated that the stiffness and the presence of sharp edges of the TM may have induced mechanical irritation to the mucosal flaps, thereby enhancing the risk of soft tissue dehiscences with consequent device exposure. ${ }^{29}$ This supposition might be corroborated by the fact that, differently from the present study, collagen membranes used to cover the TM yielded nonstatistically significant differences in terms of device exposure compared to PTFE membranes. ${ }^{21}$ Thus, the collagen membrane may act as an additional protecting layer toward the soft tissues against the TM irregularities, thereby reducing the risk of flap dehiscences, at least during the early stages of healing. This could not be confirmed in the present investigation since the titanium meshes were not covered by resorbable membranes to reduce the risk of bias.

Histological observations from the retrieved bone samples confirmed the possibility of supracrestal bone regeneration in direct contact with the titanium surface in both groups. It is worthy of note that the particulated graft appeared embedded and surrounded 
by newly formed bone, making it difficult to distinguish the limit between native bone and regenerated tissue. After 8 months of healing, it was not possible to find histological differences in the regenerated tissue quality between the two groups. The same applies to the quantitative aspect, with nonstatistically significant differences in the mean percentage of mineralized bone between the two groups. In the present study, the d-PM group revealed a mean percentage of BIC higher than the TM group. It is worth mentioning that this difference was not statistically significant between the two groups. Similarly, in a recent trial, histological and histomorphometric analyses showed no statistically significant differences in terms of newly formed bone following vertical GBR with PTFE membrane versus TM. ${ }^{22}$ In a previous study, where machined-surface mini-implants were used for histomorphometric analysis, Rocchietta et al. reported a mean BIC value of $26.62 \%$ when particulated graft was covered by a nonresorbable PTFE membrane for vertical bone augmentation. ${ }^{5}$ BIC values ranging from 25.25 to $32.80 \%$ were also discovered in a prospective study on vertical ridge augmentation by means of GBR. ${ }^{24}$

Recently, the possibility to leave the titanium grid exposed to the oral environment without jeopardizing the regenerating process has been claimed. ${ }^{25,27}$ According to these studies, removal of the TM should be contemplated in case of early exposure, namely within 3 to 4 weeks from the surgical procedure. At later stages, topical chlorhexidine gel could be used to avoid overinfection and consequent bone loss. This favorably complies with Rasia dal Polo and coworkers who found that, in most reviewed articles, late exposure was not followed by titanium mesh removal by default and did not jeopardize implant insertion in all except one study. On the other hand, a reduction of the bone gain was observed in half of the studies. These findings were in agreement with the results of the present study, where histologic analysis revealed no or limited vertical bone formation in case of TM exposure. This has also been confirmed by the intrasurgical measurements of the bone gain, varying from 0 to $1 \mathrm{~mm}$. Conversely, implant insertion could be performed in all cases, including those exhibiting TM exposures. Within the limitation of the present study, a cause-andeffect relationship between grid exposure and bone loss may be deduced both clinically and histologically, as previously defined by Proussaefs and colleagues. ${ }^{17}$ The authors reported that the slightest bone regeneration values were associated with those areas where flap dehiscence occurred. This fully agrees with other authors who found a positive correlation between TM exposure and lacking bone volume. ${ }^{30}$

A limitation of the present study is represented by the relatively limited sample size, which does not allow drawing any final conclusion. This is explained by the difficulties in recruiting patients presenting with mandibular bilateral vertical bone defects. After all, the choice of the posterior mandible as the surgical site has been made to compare the two groups in what is considered to be the most difficult and challenging anatomical area to handle. The strict inclusion criteria adopted herein can be the reason why this might be the first study, to the best of the authors' knowledge, that compared two GBR techniques in the same subject with a split-mouth approach. The small number of patients included led to a reduced power to detect small differences between the two groups from a statistical perspective. However, it is noteworthy that the results obtained in the present investigation were almost completely in accordance with those reported in previous studies with similar aims and extended populations. In view of the aforesaid, the present patient population might be considered adequate for a preliminary study and may serve as a reference to design further randomized controlled clinical trials.

\section{Conclusion}

Within the limits of the present investigation, both d-PM and TM groups showed encouraging clinical and histologic results in terms of vertical bone augmentation. In this respect, nonstatistically significant differences were observed in case of undisturbed healing. In sites exhibiting wound dehiscences, major resorption was noticed. Further clinical trials, with increased patient population based on the data reported herein, should be designed to investigate the potential variables that may influence the bone regeneration from qualitative and quantitative aspects.

\section{Clinical Significances}

Vertical bone augmentation of localized atrophic ridges can be successfully accomplished by means of nonresorbable barriers, irrespective of the type of device. The exposure of titanium mesh seems to be strictly related to a reduced vertical bone gain.

\section{ACKNOWLedgment}

This research received no external funding. Camlog Biotechnologies AG is acknowledged for donating the implants used in the present study.

\section{Ethical Approval}

The study protocol was submitted to and approved by the local institutional review board (Fondazione IRCCS Cà Granda Maggiore Policlinico Hospital Ethical Committee), with the registration number 293/12.

\section{References}

1. Beretta M, Cicciu M, Poli PP, et al. A retrospective evaluation of 192 implants placed in augmented bone: long-term follow-up study. J Oral Implantol 2015;41(6):669-674. DOI: 10.1563/aaid-joi-D-14-00123.

2. Schwartz-Arad D, Levin L, Sigal L. Surgical success of intraoral autogenous block onlay bone grafting for alveolar ridge augmentation. Implant Dent 2005;14(2):131-138. DOI: 10.1097/01. id.0000165031.33190.0d.

3. Cano J, Campo J, Moreno LA, et al. Osteogenic alveolar distraction: a review of the literature. Oral Surg Oral Med Oral Pathol Oral Radiol Endod 2006;101(1):11-28. DOI: 10.1016/j.tripleo.2005.04.015.

4. Poli PP, Beretta M, Cicciu M, et al. Alveolar ridge augmentation with titanium mesh. A retrospective clinical study. Open Dent J 2014;8:148-158. DOI: 10.2174/1874210601408010148.

5. Rocchietta I, Fontana F, Simion M. Clinical outcomes of vertical bone augmentation to enable dental implant placement: a systematic review. J Clin Periodontol 2008;35(8 Suppl.):203-215. DOI: 10.1111/j.1600-051X.2008.01271.x.

6. Esposito M, Grusovin MG, Felice $P$, et al. Interventions for replacing missing teeth: horizontal and vertical bone augmentation techniques for dental implant treatment. Cochrane Database Syst Rev 2009(4):Cd003607. DOI: 10.1002/14651858.CD003607.pub3.

7. Simion M, Jovanovic SA, Tinti C, et al. Long-term evaluation of osseointegrated implants inserted at the time or after vertical ridge augmentation. A retrospective study on 123 implants with 1-5 year follow-up. Clin Oral Implants Res 2001;12(1):35-45. DOI: 10.1034/j.1600-0501.2001.012001035.x.

8. Urban IA, Monje A, Lozada JL, et al. Long-term evaluation of peri-implant bone level after reconstruction of severely atrophic 
edentulous maxilla via vertical and horizontal guided bone regeneration in combination with sinus augmentation: a case series with 1 to 15 years of loading. Clin Implant Dent Relat Res 2017;19(1):46-55. DOI: 10.1111/cid.12431.

9. Urban IA, Lozada JL, Jovanovic SA, et al. Vertical ridge augmentation with titanium-reinforced, dense-PTFE membranes and a combination of particulated autogenous bone and anorganic bovine bone-derived mineral: a prospective case series in 19 patients. Int J Oral Maxillofac Implants 2014;29(1):185-193. DOI: 10.11607/jomi.3346.

10. Ronda M, Rebaudi A, Torelli L, et al. Expanded vs. dense polytetrafluoroethylene membranes in vertical ridge augmentation around dental implants: a prospective randomized controlled clinical trial. Clin Oral Implants Res 2014;25(7):859-866. DOI: 10.1111/clr.12157.

11. Boyne PJ. Restoration of osseous defects in maxillofacial casualities. J Am Dent Assoc 1969;78(4):767-776. DOI: 10.1016/s00028177(69)84023-7.

12. Boyne PJ, Cole MD, Stringer D, et al. A technique for osseous restoration of deficient edentulous maxillary ridges. J Oral Maxillofac Surg 1985;43(2):87-91. DOI: 10.1016/0278-2391(85)90054-0.

13. Gongloff RK, Cole M, Whitlow W, et al. Titanium mesh and particulate cancellous bone and marrow grafts to augment the maxillary alveolar ridge. Int J Oral Maxillofac Surg 1986;15(3):263-268. DOI: 10.1016/ s0300-9785(86)80083-7.

14. Maiorana C, Santoro F, Rabagliati M, et al. Evaluation of the use of iliac cancellous bone and anorganic bovine bone in the reconstruction of the atrophic maxilla with titanium mesh: a clinical and histologic investigation. Int J Oral Maxillofac Implants 2001;16(3):427-432.

15. Pieri F, Corinaldesi G, Fini M, et al. Alveolar ridge augmentation with titanium mesh and a combination of autogenous bone and anorganic bovine bone: a 2-year prospective study. J Periodontol 2008;79(11):2093-2103. DOI: 10.1902/jop.2008.080061.

16. Corinaldesi G, Pieri F, Marchetti C, et al. Histologic and histomorphometric evaluation of alveolar ridge augmentation using bone grafts and titanium micromesh in humans. J Periodontol 2007;78(8):1477-1484. DOI: 10.1902/jop.2007.070001.

17. Proussaefs $P$, Lozada J. Use of titanium mesh for staged localized alveolar ridge augmentation: clinical and histologichistomorphometric evaluation. J Oral Implantol 2006;32(5):237-247. DOI: 10.1563/1548-1336(2006)32[237:UOTMFS]2.0.CO;2.

18. Lozada J, Proussaefs P. Clinical radiographic, and histologic evaluation of maxillary bone reconstruction by using a titanium mesh and autogenous iliac graft: a case report. J Oral Implantol 2002;28(1):9-14. DOI: 10.1563/1548-1336(2002)028<0009:CRAHEO>2.3.CO;2.

19. Roccuzzo M, Ramieri G, Spada MC, et al. Vertical alveolar ridge augmentation by means of a titanium mesh and autogenous bone grafts. Clin Oral Implants Res 2004;15(1):73-81. DOI: 10.1111/j.16000501.2004.00998.x.

20. von Arx T, Hardt N, Wallkamm B. The TIME technique: a new method for localized alveolar ridge augmentation prior to placement of dental implants. Int J Oral Maxillofac Implants 1996;11(3): 387-394

21. Cucchi A, Vignudelli E, Napolitano A, et al. Evaluation of complication rates and vertical bone gain after guided bone regeneration with non-resorbable membranes versus titanium meshes and resorbable membranes. A randomized clinical trial. Clin Implant Dent Relat Res 2017;19(5):821-832. DOI: 10.1111/ cid.12520.

22. Cucchi A, Sartori M, Parrilli A, et al. Histological and histomorphometric analysis of bone tissue after guided bone regeneration with nonresorbable membranes vs resorbable membranes and titanium mesh. Clin Implant Dent Relat Res 2019;21(4):693-701. DOI: 10.1111/ cid.12814.

23. O'Leary TJ, Drake RB, Naylor JE. The plaque control record. J Periodontol 1972;43(1):38. DOI: 10.1902/jop.1972.43.1.38.

24. Fontana F, Santoro F, Maiorana C, et al. Clinical and histologic evaluation of allogeneic bone matrix versus autogenous bone chips associated with titanium-reinforced e-PTFE membrane for vertical ridge augmentation: a prospective pilot study. Int J Oral Maxillofac Implants 2008;23(6):1003-1012.

25. Rasia-dal Polo M, Poli PP, Rancitelli D, et al. Alveolar ridge reconstruction with titanium meshes: a systematic review of the literature. Med Oral Patol Oral Cir Bucal 2014;19(6):e639-e646. DOI: 10.4317/medoral.19998.

26. Tinti C, Parma-Benfenati S, Polizzi G. Vertical ridge augmentation: what is the limit? Int J Periodontics Restorative Dent 1996;16(3): 220-229.

27. Ricci L, Perrotti V, Ravera L, et al. Rehabilitation of deficient alveolar ridges using titanium grids before and simultaneously with implant placement: a systematic review. J Periodontol 2013;84(9):1234-1242. DOI: 10.1902/jop.2012.120314.

28. Fontana F, Maschera E, Rocchietta I, et al. Clinical classification of complications in guided bone regeneration procedures by means of a nonresorbable membrane. Int J Periodontics Restorative Dent 2011;31(3):265-273.

29. Watzinger F, Luksch J, Millesi W, et al. Guided bone regeneration with titanium membranes: a clinical study. Br J Oral Maxillofac Surg 2000;38(4):312-315. DOI: 10.1054/bjom.1999.0228.

30. Lizio G, Corinaldesi G, Marchetti C. Alveolar ridge reconstruction with titanium mesh: a three-dimensional evaluation of factors affecting bone augmentation. Int J Oral Maxillofac Implants 2014;29(6):13541363. DOI: $10.11607 /$ jomi.3417. 